\title{
Status of Aqua MODIS On-orbit Calibration and Characterization
}

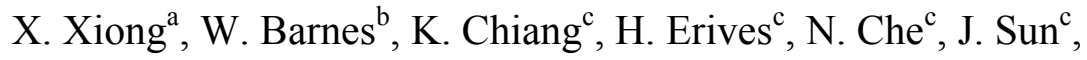 \\ A. Isaacman ${ }^{\mathrm{d}}$, and V. Salomonson ${ }^{\mathrm{a}}$ \\ ${ }^{a}$ Earth Sciences Directorate, NASA/GSFC, Greenbelt, MD 20771 \\ ${ }^{b}$ University of Maryland, Baltimore County, Baltimore, MD 21250 \\ ${ }^{\mathrm{c} S}$ Science Systems and Applications, Inc., 10210 Greenbelt Rd., Lanham, MD 20706 \\ ${ }^{\mathrm{d}}$ Science Applications International Corp., 7501 Forbes Blvd., Seabrook, MD 20706
}

\begin{abstract}
The MODIS Flight Model 1 (FM1) has been in operation for more than two years since its launch onboard the NASA's Earth Observing System (EOS) Aqua spacecraft on May 4, 2002. The MODIS has 36 spectral bands: 20 reflective solar bands (RSB) with center wavelengths from 0.41 to $2.2 \mu \mathrm{m}$ and 16 thermal emissive bands (TEB) from 3.7 to $14.5 \mu \mathrm{m}$. It provides the science community observations (data products) of the Earth's land, oceans, and atmosphere for a board range of applications. Its primary on-orbit calibration and characterization activities are performed using a solar diffuser (SD) and a solar diffuser stability monitor (SDSM) system for the RSB and a blackbody for the TEB. Another on-board calibrator (OBC) known as the spectro-radiometric calibration assembly (SRCA) is used for the instrument's spatial (TEB and RSB) and spectral (RSB only) characterization. We present in this paper the status of Aqua MODIS calibration and characterization during its first two years of on-orbit operation. Discussions will be focused on the calibration activities executed on-orbit in order to maintain and enhance the instrument's performance and the quality of its Level 1B (L1B) data products. We also provide comparisons between Aqua MODIS and Terra MODIS (launched in December, 1999), including their similarity and difference in response trending and optics degradation. Existing data and results show that Aqua MODIS bands $8(0.412 \mu \mathrm{m})$ and $9(0.443 \mu \mathrm{m})$ have much smaller degradation than Terra MODIS bands 8 and 9. The most noticeable feature shown in the RSB trending is that the mirror side differences in Aqua MODIS are extremely small and stable $(<0.1 \%)$ while the Terra MODIS RSB trending has shown significant mirror side difference and wavelength dependent degradation. The overall stability of the Aqua MODIS TEB is also better than that of the Terra MODIS during their first two years of on-orbit operation.
\end{abstract}

Keywords: Aqua, Terra, MODIS, solar diffuser, SRCA, blackbody, calibration, radiometry, uncertainty

\section{INTRODUCTION}

Two nearly identical copies of the Moderate Resolution Imaging Spectroradiometer (MODIS), the Protoflight Model (PFM) and the Flight Model 1 (FM1), were designed and built by Raytheon/Santa Barbara Remote Sensing (SBRS) for the NASA's Earth Observing System (EOS) missions ${ }^{1-3}$. The MODIS instrument was designed and developed based on the desire of the science community to improve and extend existing (heritage) sensors' capabilities in terms of spectral (band and bandwidth) coverage, spatial resolutions, and temporal scales. It has 36 spectral bands with wavelengths from 0.41 to $14.5 \mu \mathrm{m}$. It makes measurements at three different nadir spatial resolutions: $250 \mathrm{~m}$ for bands $1-2$ (40 detectors/band), $500 \mathrm{~m}$ for bands 3-7 (20 detectors/band), and $1000 \mathrm{~m}$ for bands 8-36 (10 detectors/band). Bands 1-19, and 26 are the reflective solar bands (RSB) and the others are the thermal emissive bands (TEB). The 36 spectral bands are distributed, according to their wavelengths, on four focal plane assemblies (FPAs): visible (VIS), near infrared (NIR), short- and mid-wave infrared (SMIR), and long-wave infrared (LWIR). MODIS is a passive cross-track scanning radiometer with a wide field-of-view (FOV) of $\pm 55^{\circ}$ about instrument nadir and uses a double-sided paddle wheel scan 
mirror that rotates at $20.3 \mathrm{rpm}$. At a nominal orbiting altitude of $705 \mathrm{~km}$, each scan of 1.478 seconds produces a swath of $10 \mathrm{~km}$ (nadir) in the along track direction and $2330 \mathrm{~km}$ in the along scan direction, resulting in near-global coverage of the Earth every 2 days.

The MODIS PFM was successfully launched on December 18, 1999 on-board the EOS Terra spacecraft and the FM1 on May 04, 2002 on-board the EOS Aqua spacecraft, both from Vandenberg Air Force Base in California. There are more than 40 science products that are generated from MODIS observations ${ }^{4-8}$. Most of them start from the MODIS Level 1B data products: radiometrically calibrated and geo-located top of the atmosphere (TOA) radiance (RSB and TEB) and reflectance (RSB). Together, Terra and Aqua MODIS have produced more than 78 months of data produces. Table 1 lists several key design parameters of the MODIS spectral bands including their bandwidths, specified typical radiances, signal to noise ratio (SNR) or noise equivalent temperature difference (NEdT) requirements, and their primary science applications.

Table 1: Summary of the MODIS spectral bands (primary applications, bandwidth, typical spectral radiance and required SNR or NEdT).

\begin{tabular}{|c|c|c|c|c|c|c|c|c|c|}
\hline Primary Use & Band $^{1}$ & $\begin{array}{l}\text { Bandwidth } \\
(\mathrm{nm})\end{array}$ & $\begin{array}{l}\text { Spectral } \\
\text { Radiance }^{2}\end{array}$ & $\begin{array}{l}\text { Required } \\
\text { SNR }\end{array}$ & Primary Use & Band $^{1}$ & $\begin{array}{c}\text { Bandwidth } \\
(\mu \mathrm{m})\end{array}$ & \begin{tabular}{|c|} 
Spectral \\
Radiance $^{2}$ \\
\end{tabular} & $\begin{array}{l}\text { Required } \\
\mathrm{NE} \Delta \mathrm{T}(\mathrm{K})\end{array}$ \\
\hline \multirow{2}{*}{$\begin{array}{l}\text { Land/Cloud/Aerosols } \\
\text { Boundaries }\end{array}$} & 1 & $620-670$ & 21.8 & 128 & \multirow{4}{*}{$\begin{array}{l}\text { Surface/Cloud } \\
\text { Temperature }\end{array}$} & 20 & $3.660-3.840$ & $0.45(300 K)$ & 0.05 \\
\hline & 2 & $841-876$ & 24.7 & 201 & & 21 & $3.929-3.989$ & $2.38(335 K)$ & 0.2 \\
\hline \multirow{5}{*}{$\begin{array}{l}\text { Land/Cloud/Aerosols } \\
\text { Properties }\end{array}$} & 3 & $459-479$ & 35.3 & 243 & & 22 & $3.929-3.989$ & $0.67(300 \mathrm{~K})$ & 0.07 \\
\hline & 4 & $545-565$ & 29 & 228 & & 23 & $4.020-4.080$ & $0.79(300 \mathrm{~K})$ & 0.07 \\
\hline & 5 & $1230-1250$ & 5.4 & 74 & \multirow{2}{*}{$\begin{array}{l}\text { Atmospheric } \\
\text { Temperature }\end{array}$} & 24 & $4.433-4.498$ & 0.17 (250K) & 0.25 \\
\hline & 6 & $1628-1652$ & 7.3 & 275 & & 25 & $4.482-4.549$ & $0.59(275 K)$ & 0.25 \\
\hline & 7 & $2105-2155$ & 1 & 110 & \multirow{3}{*}{$\begin{array}{l}\text { Cirrus Clouds Water } \\
\text { Vapor }\end{array}$} & 26 & $1.360-1.390$ & 6 & 150 (SNR) \\
\hline \multirow{9}{*}{$\begin{array}{l}\text { Ocean Color/ } \\
\text { Phytoplankton/ } \\
\text { Biogeochemistry }\end{array}$} & 8 & $405-420$ & 44.9 & 880 & & 27 & $6.535-6.895$ & $1.16(240 K)$ & 0.25 \\
\hline & 9 & $438-448$ & 41.9 & 838 & & 28 & $7.175-7.475$ & $2.18(250 K)$ & 0.25 \\
\hline & 10 & $483-493$ & 32.1 & 802 & Cloud Properties & 29 & $8.400-8.700$ & $9.58(300 \mathrm{~K})$ & 0.05 \\
\hline & 11 & $526-536$ & 27.9 & 754 & Ozone & 30 & $9.580-9.880$ & $3.69(250 \mathrm{~K})$ & 0.25 \\
\hline & 12 & $546-556$ & 21 & 750 & \multirow{2}{*}{$\begin{array}{l}\text { Surface/Cloud } \\
\text { Temperature }\end{array}$} & 31 & $10.780-11.280$ & $9.55(300 \mathrm{~K})$ & 0.05 \\
\hline & 13 & $662-672$ & 9.5 & 910 & & 32 & $11.770-12.270$ & $8.94(300 \mathrm{~K})$ & 0.05 \\
\hline & 14 & $673-683$ & 8.7 & 1087 & \multirow[t]{4}{*}{ Cloud Top Altitude } & 33 & $13.185-13.485$ & $4.52(260 K)$ & 0.25 \\
\hline & 15 & $743-753$ & 10.2 & 586 & & 34 & $13.485-13.785$ & 3.76 (250K) & 0.25 \\
\hline & 16 & $862-877$ & 6.2 & 516 & & 35 & $13.785-14.085$ & $3.11(240 K)$ & 0.25 \\
\hline \multirow{3}{*}{$\begin{array}{l}\text { Atmospheric Water } \\
\text { Vapor }\end{array}$} & 17 & $890-920$ & 10 & 167 & & 36 & $14.085-14.385$ & $2.08(220 K)$ & 0.35 \\
\hline & 18 & $931-941$ & 3.6 & 57 & \multirow{2}{*}{\multicolumn{5}{|c|}{$\begin{array}{l}{ }^{1} \text { Spatial resolution: } 250 \mathrm{~m}(\mathrm{~B} 1-2), 500 \mathrm{~m} \text { (B3-7), 1000m (B8-36) } \\
{ }^{2} \text { Spectral Radiance unit: W/m } \mathrm{m}^{2}-\mu \mathrm{m}-\mathrm{sr}\end{array}$}} \\
\hline & 19 & $915-965$ & 15 & 250 & & & & & \\
\hline
\end{tabular}

In this paper, we provide an overview of the Aqua MODIS on-orbit calibration and characterization activities since its launch and summarize the instrument's radiometric, spatial, and spectral performance, including comparisons with its predecessor, the Terra MODIS. This paper serves as an update to our previous presentations that included detailed descriptions of the Aqua MODIS characteristics and its early on-orbit results ${ }^{9-11}$. The results derived from the first two years of Aqua MODIS on-orbit operation and observations show that the TEB detectors' responses are very stable with small changes or variations of less than $0.5 \%$ per year. The responses of the RSB, partially due to optics degradation, are spectral band (wavelength) dependent. These changes are determined from instrument on-orbit calibration and characterization and therefore captured in the calibration algorithms in order to maintain data product quality. Except for a few problems identified pre-launch, such as inoperable detectors in band 6 and band-to-band registration (BBR) concerns, the Aqua MODIS has been performing according its design characteristics and meeting its calibration accuracy requirements ( $1 \sigma$ at the typical spectral radiance): $\pm 2 \%$ for the RSB reflectance factors and $\pm 5 \%$ for the RSB radiance; 
and $\pm 1 \%$ for the TEB radiance except $\pm 0.75 \%$ for band 20 at $3.75 \mu \mathrm{m}$ and $\pm 0.5 \%$ for bands 31 and 32 at $11 \mu \mathrm{m}$ and $12 \mu \mathrm{m}$. Band 21 is used for fire detection with very low gain and its accuracy requirement is $\pm 10 \%$. Up to now, the on-orbit shifts of the RSB spectral bands' center wavelengths are very small (less than $0.5 \mathrm{~nm}$ ). Although the Aqua MODIS bandto-band registration (BBR) is out of specification when registering bands on the warm FPAs (VIS and NIR) to those on the cold FPAs (MWIR and LWIR), there has been no obvious change in BBR on-orbit.

\section{OPERATIONAL EVENTS AND CALIBRATION ACTIVITIES}

The Aqua MODIS was launched on May 04, 2002. The instrument nadir aperture door was opened on June 24, 2002 to start the Earth scene observations (the first light). Except for three spacecraft induced safe-hold events during the initial on-orbit check-out period (launch +120 days) that resulted in minor data gaps, Aqua MODIS instrument has been operating smoothly for more than two years, continuously producing quality data for the science community and other users around the world. Table 2 provides a list of the major operational events of the Aqua spacecraft and the MODIS instrument during the first two years, including unexpected safe hold events that impacted data continuity and calibration quality. Unlike Terra MODIS, which has operated in a number of different configurations listed in Table 3 (note: several changes purposely made to the short- and mid-wave focal plane voltage bias are not included), Aqua MODIS has been using the same operational configuration (B-side) since launch. Because of this, the Aqua MODIS onorbit calibration and characterization is much simpler than that of Terra MODIS.

Table 2: First two years of Aqua spacecraft and MODIS Flight Mode 1 (FM1) operational events since their launch on May 04, 2002.

\begin{tabular}{|l|l|l|}
\hline \multicolumn{1}{|c|}{ Date } & \multicolumn{1}{c|}{ Events } & \multicolumn{1}{c|}{ Description } \\
\hline May 4, 2002 & Launch & Launched successfully \\
\hline Jun 7, 2002 & Science Mode & MODIS started science mode on B-side \\
\hline Jun 24, 2002 & Nadir Door Open & Aqua MODIS First Light \\
\hline Jun 27, 2002 & S/C Safe Hold & Aqua spacecraft single event upset (SEU) \\
MODIS returned to science mode on July 2
\end{tabular}

Table 3: First two years of Terra spacecraft and MODIS Proto-flight Model (PFM) operational events since their launch on December 18, 1999.

\begin{tabular}{|l|l|l|}
\hline \multicolumn{1}{|c|}{ Date } & \multicolumn{1}{c|}{ Events } & \multicolumn{1}{c|}{ Description } \\
\hline Dec 18,1999 & Launch & Launched successfully \\
\hline Feb 13,2000 & Science Mode & MODIS started science mode on A-side \\
\hline Feb 24, 2000 & Nadir Door Open & Terra MODIS First Light \\
\hline June 2000 & CFPA Lost Control & Icing on the radiative cooler surface \\
\hline Aug 5, 2000 & Formatter Anomaly & MODIS entered standby mode then safe mode \\
\hline Aug 8,2000 & Outgas & Turned on outgas heater for two days \\
\hline Aug 19,2000 & Science Mode & MODIS returned to science mode on A-side \\
\hline Oct 30, 2000 & B-side Electronics & Transitioned to science mode on B-side \\
\hline Jun 15,2001 & PS2 Anomaly & Powered supply 2 (B-side) shut down \\
\hline Jul 2, 2001 & A-side Electronics & Returned to science mode on A-side with PS1 \\
\hline
\end{tabular}

The MODIS on-board calibrators include a solar diffuser (SD), a spectroradiometric calibration assembly (SRCA), and a blackbody (BB). In addition, the sensor's view through the space view (SV) port provides instrument background and offset information. Another on-board device, the solar diffuser stability monitor (SDSM), is used to track the SD on-orbit 
degradation. Normally, the MODIS thermal emissive bands are calibrated on a scan-by-scan basis using the OBC vgroove blackbody (BB) data and the reflective solar bands are calibrated bi-weekly using the calibration coefficients from the SD/SDSM observations. MODIS on-orbit radiometric calibration activities consist of SD/SDSM observations (weekly first year and bi-weekly afterwards) for the RSB and BB warm-up and cool-down cycle (quarterly) for the TEB. Normally the SD door is closed to minimize direct solar exposure of the SD panel and the BB is set at 285K (for Aqua MODIS). Because of a dropped command during a scheduled SD calibration, the SD door was left open August 9-14, 2002 , resulting in a noticeable increase in degradation of the SD during this period. The SRCA is capable of operating in three different modes: radiometric (monthly), spatial (bi-monthly) and spectral (quarterly). Shortly after launch, one of the SRCA 10W lamps was found to be unstable based on its on-orbit observations and was replaced by its backup. The electronic calibration (ECAL), used to check the electronics' function, is performed bi-monthly. Other activities include lunar observations (9-10 times per year) through the space view (SV) port via spacecraft roll maneuvers and special $\mathrm{SD} / \mathrm{SDSM}$ operations during spacecraft yaw maneuvers performed to derive the SD screen vignetting function. The latter is critical to the RSB high-gain bands calibration.

There were a number of improvements made in the Aqua MODIS (Flight Model 1) design and characterization based on lessons learned from the Terra MODIS (Protoflight Model), including reduction of the SWIR bands (bands 5-7, 26) electronic crosstalk ${ }^{12}$. To further reduce SWIR cross-talk, a correction algorithm used for Terra MODIS is also applied in the Aqua L1B code ${ }^{13}$. The correction coefficients are derived and updated as needed from specially scheduled nighttime observations with the instrument operated in the daytime mode since normally there is no RSB Earth view (EV) data collected during spacecraft nighttime. This special activity allows the EV sector data to be collected for the reflective solar bands during orbit nighttime.

\section{ON-ORBIT CALIBRATION ALGORITHMS}

The primary MODIS Level 1B (L1B) product for the RSB is top of the atmosphere (TOA) reflectance and for the TEB, TOA radiance. The RSB radiance product is derived from the reflectance factor. MODIS calibration is performed and applied for each band, detector, sub-frame (for sub-kilometer bands 1-7), and mirror side (BSDM) with specified calibration accuracy requirements ( $1 \sigma$ at the typical spectral radiance) of $\pm 2 \%$ for the RSB reflectance factor and $\pm 5 \%$ for the RSB radiance and $\pm 1 \%$ for the TEB radiance except $\pm 0.75 \%$ for band 20 at $3.75 \mu \mathrm{m}, \pm 10 \%$ for band 21 at $3.96 \mu \mathrm{m}$ (low gain for fire detection), and $\pm 0.5 \%$ for bands 31 and 32 at $11 \mu \mathrm{m}$ and $12 \mu \mathrm{m}$ (for sea surface temperature). The radiometric calibration is provided by the SD/SDSM for the RSB and by the $\mathrm{BB}$ for the TEB. On-orbit spectral (RSB) and spatial (RSB and TEB) characterization is performed by the SRCA.

All the on-board calibrators were calibrated and characterized pre-launch using ground-based equipment with higher accuracy and precision. The functions of the OBCs include maintaining calibration scales, tracking and removing if necessary any changes that would impact on-orbit calibration quality. In the following, a very brief description is given for each of the key calibration and characterization activities. The on-orbit performance derived from these activities will be presented in the next section.

\subsection{RSB calibration}

For each Earth view (EV) pixel, the RSB reflectance factor, $\rho_{\mathrm{EV}} \cos \left(\theta_{\mathrm{EV}}\right)$, is related to the calibration coefficient $\mathrm{m}_{1}$ determined from $\mathrm{SD} / \mathrm{SDSM}$ calibration and the sensor's $\mathrm{EV}$ response $\mathrm{dn}_{\mathrm{EV}} *$ by a linear equation,

$$
\rho_{E V} \cos \left(\theta_{E V}\right)=m_{1} \cdot d n_{E V}^{*} \cdot d_{E S}^{2}
$$

where $\theta_{\mathrm{EV}}$ is the Earth scene solar zenith angle, $\mathrm{dn}_{\mathrm{EV}} \mathrm{v}^{*}$ is the sensor's digital response corrected for instrument temperature, background, and scan angle effects, and $\mathrm{d}_{\mathrm{ES}}$ is the Earth-Sun distance at the time of the EV observation normalized to $1 \mathrm{AU}$.

The RSB calibration coefficients $\mathrm{m}_{1}$ are computed using the same approach by including the sensor's response to the solar illuminated SD, the SD bi-directional reflectance factor (BRF), and the on-orbit SD BRF degradation. For high gain bands (bands 8-16), the SD screen vignetting function is also applied. In addition, lunar observations and SRCA 
radiometric calibration data sets are used to support RSB calibration, providing valuable information for tracking changes in the sensor's response versus scan angle (RVS) since these measurements are made at different angle of incidence (AOI) to the scan mirror. The RSB calibration coefficients are updated regularly in the L1B algorithm through Look-up Tables (LUTs). Details of the RSB calibration algorithms including the equations used to compute the calibration coefficients and SD degradation can be found in our previous presentations ${ }^{14,15}$.

\subsection{TEB calibration}

There are no sub-frames in the thermal emissive bands. The EV path radiance, including the EV TOA radiance, $\mathrm{L}_{\mathrm{EV}}$, and a known contribution from the MODIS scan mirror thermal emission, $\mathrm{L}_{\mathrm{SM}}$, is computed for each band, detector, and mirror side using a quadratic algorithm,

$$
R V S_{E V} \cdot L_{E V}+\left(R V S_{S V}-R V S_{E V}\right) \cdot L_{S M}=a_{0}+b_{1} \cdot d n_{E V}+a_{2} \cdot d n_{E V}^{2}
$$

where $\mathrm{dn}_{\mathrm{EV}}$ is the detector's digital response to the EV after subtracting the response to the SV. The offset term $\mathrm{a}_{0}$ and nonlinear term $\mathrm{a}_{2}$ of the quadratic calibration coefficients were determined from pre-launch calibrations and are updated on-orbit using BB warm-up and cool-down cycle data.

The linear coefficient $b_{1}$, not $a_{1}$ to emphasize the on-orbit calibration process, is determined from the detector's response to the on-board BB each scan. The scan mirror (SM) contribution term in the EV path radiance is due to the response versus scan angle (RVS) difference at different angle of incidence (AOI) to the scan mirror between the EV and the $\mathrm{SV}^{14,16}$. Aqua MODIS TEB RVS was derived from pre-launch system level measurements and provided to the L1B through LUTs.

\subsection{Spectral characterization}

The spectral and spatial characterization of the instrument is provided by the SRCA. In the spectral mode, the SRCA is essentially a monochromator. It has internal wavelength calibration capability with its own light sources, calibration and reference photodiodes. The MODIS detectors' responses to the SRCA illumination, after removing the SRCA source spectrum, yields the channels' (detector plus optics) relative spectral responses (RVS),

$$
R S R(\lambda)=\frac{d n_{S R C A_{-} N o m}(\lambda)}{\max \left\{d n_{\mathrm{SRCA}_{-} \mathrm{Nom}}(\lambda)\right\}}
$$

where the wavelength $\lambda$ is related to the SRCA monochromator grating position and $\operatorname{dn}_{\text {SRCA Nom }}(\lambda)$ is the channel's response to the SRCA monochromator source at a given wavelength or grating position subtracted from the SV offset and corrected for the source spectrum.

Due to the limitations of SRCA internal source, the spectral response measured on-orbit is only portion of the in-band response for the reflective solar bands. Consecutive on-orbit measurements can adequately track changes of the MODIS detectors' spectral responses and shifts in their center wavelengths ${ }^{17,18}$.

\subsection{Spatial characterization}

In the spatial mode, the grating of the monochromator in the SRCA is replaced with a plain mirror. Thus the monochromator in the spectral mode becomes a relay collimator in the spatial mode. Two reticles of different shapes are used to measure the changes in band-to-band registration (BBR), one for the along scan direction and the other for the along track direction. Additional data sets are collected with five different phase delays (step size of $0.2 \mathrm{~km}$ of a $1.0 \mathrm{~km}$ IFOV) in the start of the sampling period, which is equivalent to moving the reticle step by step.

In general the centroid of the response determines the band or detector's position $<\mathrm{x}>$, 


$$
<x>=\frac{\sum x \cdot d n_{S R C A}}{\sum d n_{S R C A}}
$$

where $\mathrm{dn}_{\mathrm{SRCA}}$ is the detector's response to the SRCA source (through the reticle) in the spatial mode with the SV offset subtracted. For the along scan direction the centroid can be determine for each detector. The $\mathrm{x}$ in Eqn. 4 is related to the sample frame number and phase delay applied in the measurements. For sub-kilometer bands, the sub-frames can also be included. The changes in centroid values (averaged over all detectors in a band) correspond to the changes in BBR. Band 1 on the NIR foal plane is used as the reference.

The along track BBR measurements are more complicated since MODIS is a crosstrack scanning radiometer. The along track reticle has two stepped openings for the purpose of producing the equivalence of a knife- edge srepped in the along track direction. To increase the number of useful data samples, responses of all detectors at selected frames are used in determining the along track $\mathrm{BBR}^{19-21}$.

\subsection{Special considerations in the L1B algorithm}

Except for a few minor differences, both Aqua and Terra MODIS use nearly the same calibration algorithms in deriving the L1B data products. These differences are

(1) The Aqua SWIR bands thermal leak correction uses band 25 as the sending band (band 28 is used in Terra).

(2) Fixed calibration coefficients are used for B33, 35, and 36 when the BB temperatures are above their saturation limits.

(3) There is no PC crosstalk correction applied in the Aqua MODIS calibration algorithm.

\section{ON-ORBIT PERFORMANCE}

In this section, the overall performance of Aqua MODIS is presented, including the time series of response stability for the reflective solar bands and thermal emissive bands and on-orbit changes of the spectral and spatial characterization parameters, including center wavelengths and band-to-band registration. The similarities and differences between Aqua MODIS and Terra MODIS are illustrated by comparing their performance in the first two years starting from the "first light" on February 24, 2000 for Terra MODIS and June 24, 2002 for Aqua MODIS.

\subsection{RSB calibration performance}

Using the on-board SD/SDSM, the RSB calibration tracks changes in detector response, electronics gain, and optics degradation. Since the calibration is at an end-to-end system level, one cannot separate the changes due to individual components. The overall changes are illustrated by the calibration coefficient trending in Figure 1 for both Aqua MODIS (left) and Terra MODIS (right) RSB. Bands 3, 8, 9, 10, and 17 are used as examples of the response changes (averaged over all detectors; mirror side 1) during each sensor's first two-years of on-orbit operation.

Aqua MODIS has been in the same operational configuration since launch. However, during the first six months normal operations were interrupted three times due to spacecraft safe-hold events (Table 2). These put MODIS into its safe mode and resulted in minor data gaps. The vertical lines in the plot correspond to these safe hold events. Sometimes, these events can impact the sensor's response. In comparison, Terra MODIS has changed its major operational configurations several times (Table 3) during its first two years of on-orbit operation. The sharp increase and decrease in the Terra RSB response trending are due to the changes of instrument configuration from the A-side electronics to the Bside and then back to A-side. The overall results show that the Aqua MODIS visible bands $8(0.412 \mu \mathrm{m})$ and $9(0.443 \mu \mathrm{m})$ have much smaller degradation than the Terra MODIS bands 8 and 9. The Terra MODIS RSB trending shows a strong wavelength dependent degradation. The purpose of the calibration is to track and correct changes in order to maintain data quality and to assure science product continuity.

For the same bands the ratios of their mirror side responses are presented in Figure 2 for both Terra and Aqua MODIS. Obviously the degradation has little mirror side difference in Aqua MODIS, indicating that both mirror sides have been 
changing at the same rate. On the other hand, the change of the mirror side difference has been quite large in the Terra MODIS visible spectral region.
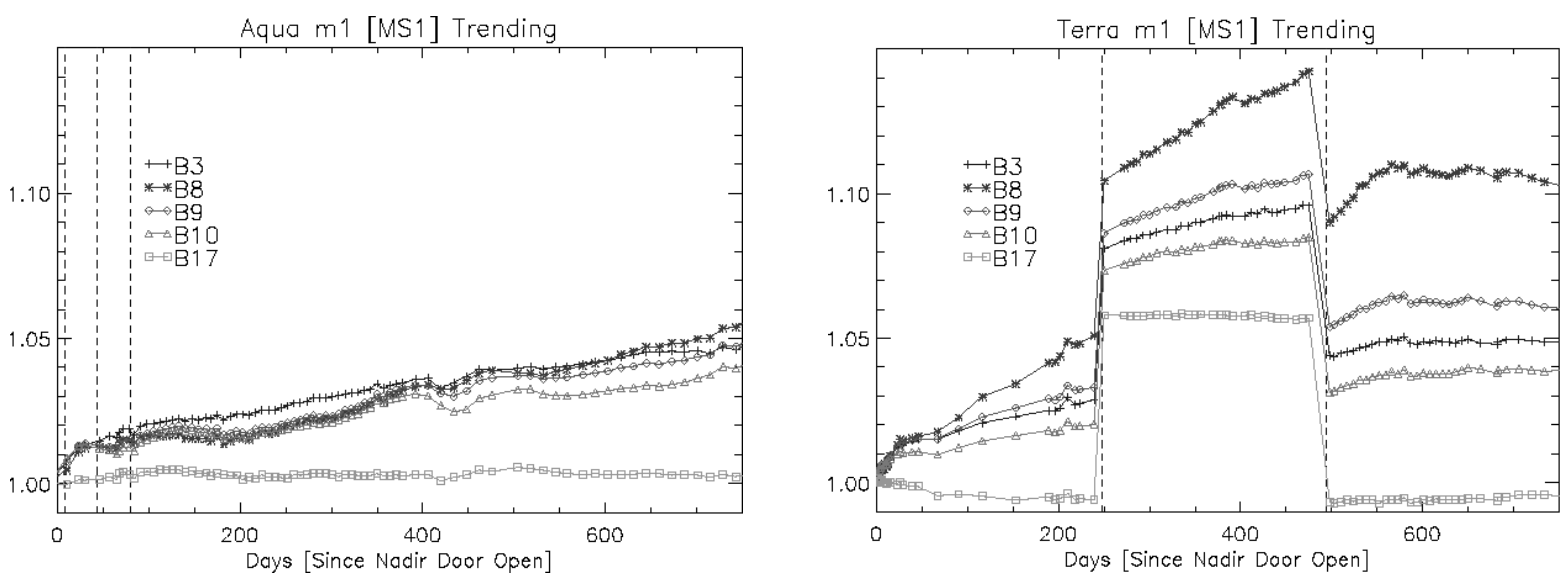

Figure 1: Reflective solar bands (3, 8, 9, 10, and 17) normalized response (averaged over all detectors in each band, mirror side 1 only) vs. days: Aqua MODIS starting from June 24, 2002 (left) and Terra MODIS starting from February 24, 2000 (right).
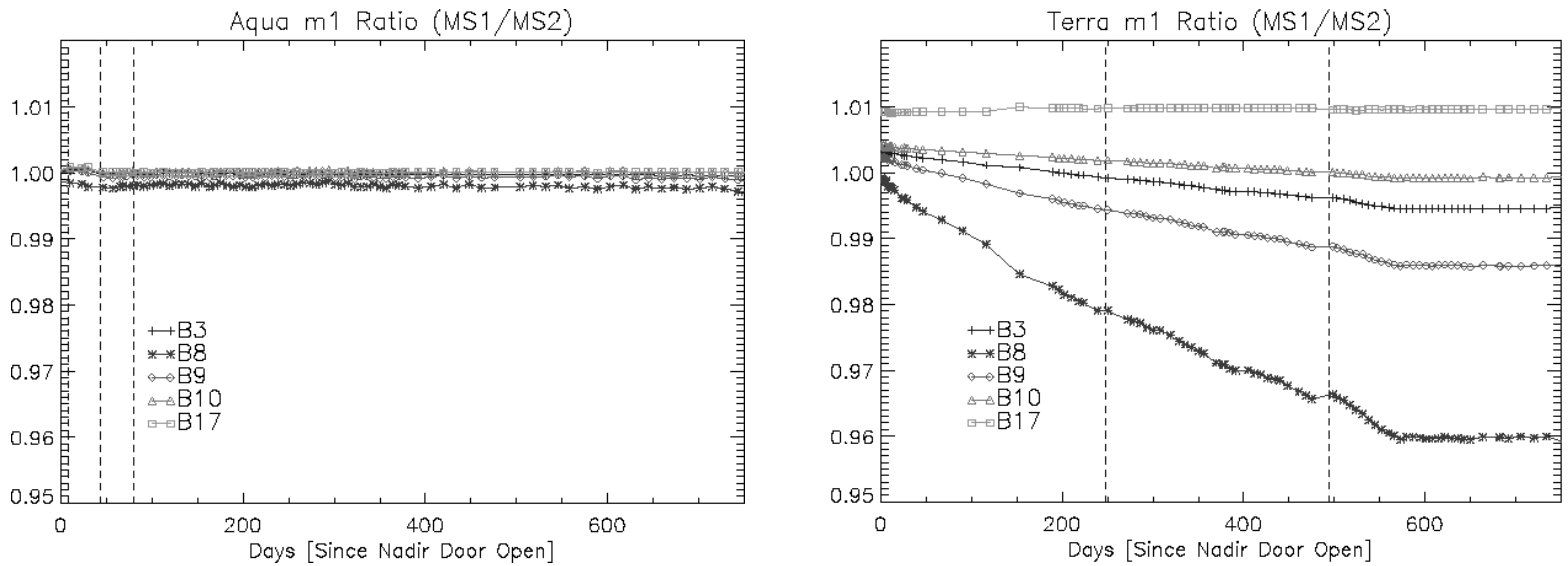

Figure 2: Reflective solar bands $(3,8,9,10$, and 17) response ratio of mirror side 1 to mirror side 2 vs. days: Aqua MODIS starting from June 24, 2002 (left) and Terra MODIS starting from February 24, 2000 (right).

\subsection{TEB calibration performance}

The Aqua MODIS on-orbit calibrations have shown excellent stability for most of the TEB detectors. This is illustrated by the trending of the TEB calibration coefficients or detectors' responses in Figures 3 and 4. Bands 31 and 35 (detectors 3,4 , and 9) are used as examples. The linear coefficient is the dominant term in the TEB calibration algorithm. Its stability reflects the overall system level performance. Since only one operational configuration has been used in Aqua MODIS, the response trending is extremely smooth. The variation of the response is less than $0.5 \% /$ year. Some of the small changes are due to the spacecraft reset events that happened at the beginning of the mission. The same TEB bands and detectors from Terra MODIS are included for comparison. The Terra TEB response trending is also very stable except during the period when the radiative cooler lost control of the FPAs' temperatures. Normally the cold FPAs are controlled at $83^{\circ} \mathrm{K}$. The TEB detectors' responses are a function of the FPAs' temperature. The other obvious discontinuities are due to changes of the operational configurations (see Table 3) as different electronics configurations have slightly different signal amplifications.

Figures 5 and 6 provide the mirror side differences over the same period for MODIS bands 31 and 35 (detectors 3, 4, and 9 ). The difference is extremely small (less than $\pm 0.025 \%$ ) and stays nearly constant for the Aqua MODIS TEB. Because 
different operational configurations were used in Terra MODIS, the response ratio of the two mirror sides shows noticeable mirror-side correlated noise (less than $\pm 0.1 \%$ for band 31 and less than $\pm 0.5 \%$ for band 35 ) that are primarily due to the different electronics configurations. Note that most of mirror side differences (Figures 5 and 6) are calibrated out since the TEB calibration algorithm treats each mirror side separately.
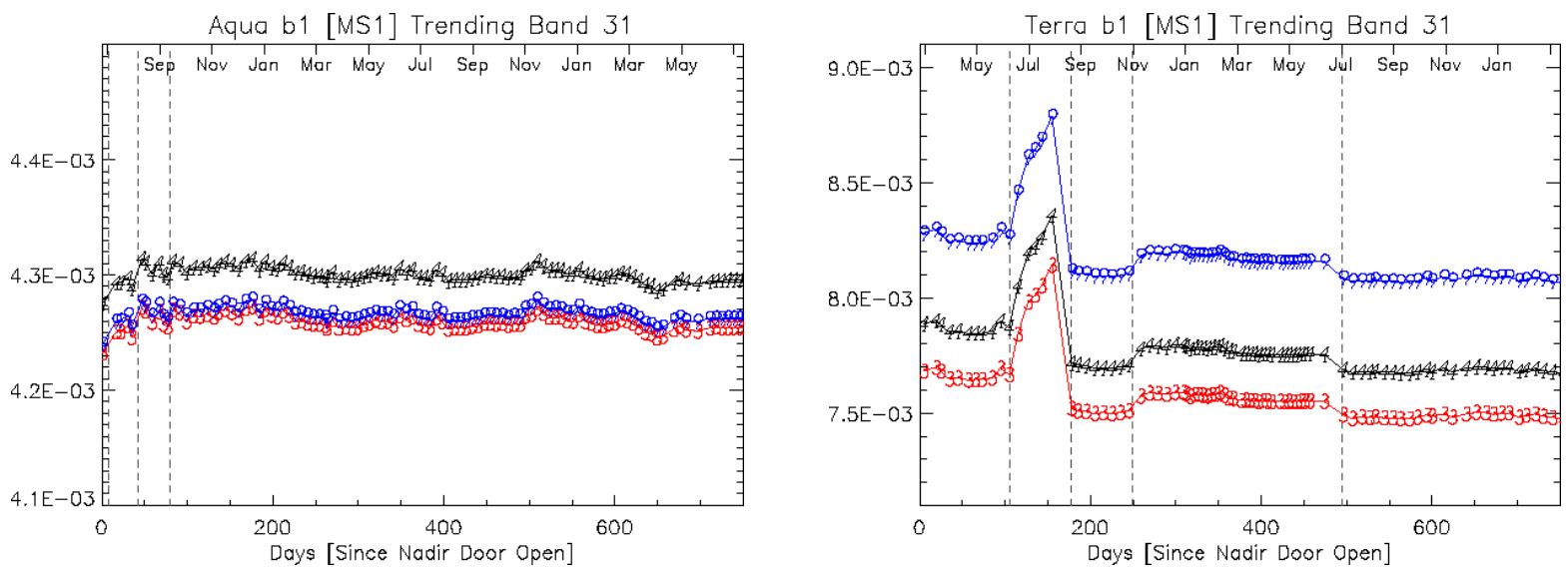

Figure 3: Aqua and Terra MODIS B31 (detectors 3, 4, and 9) response or calibration coefficient b1 vs. days.
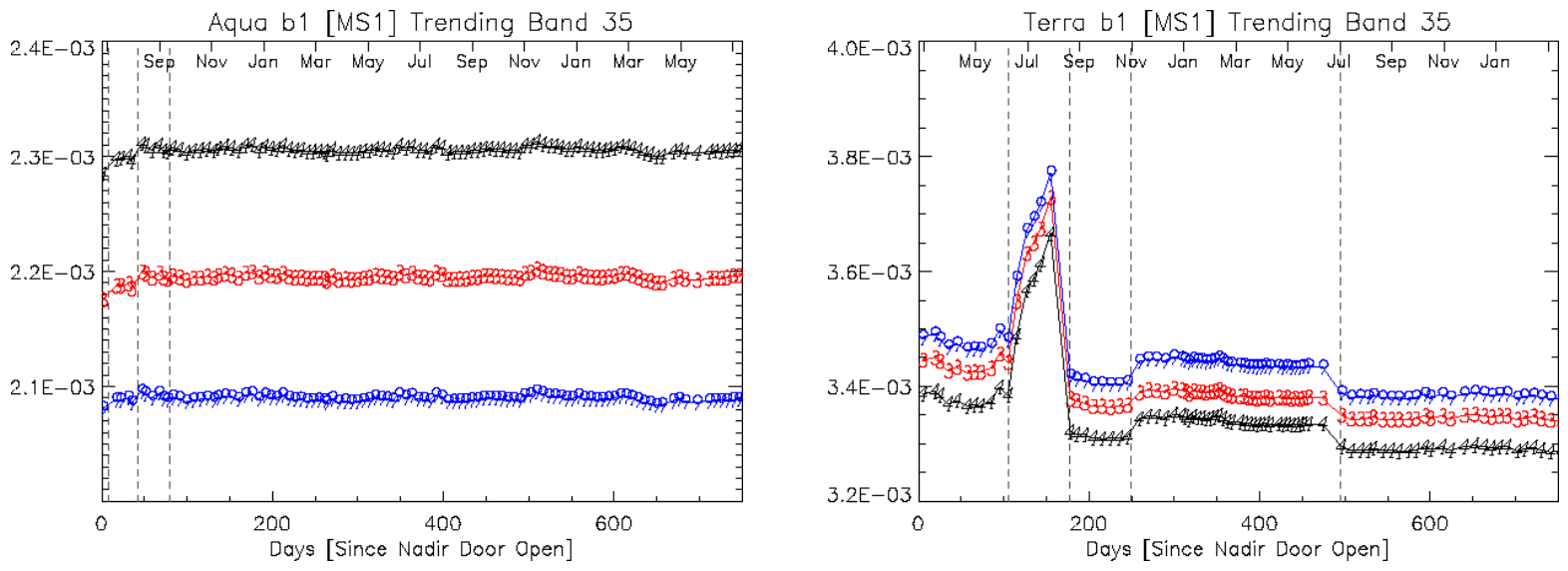

Figure 4: Aqua and Terra MODIS B35 (detectors 3, 4, and 9) response or calibration coefficient b1 vs. days.
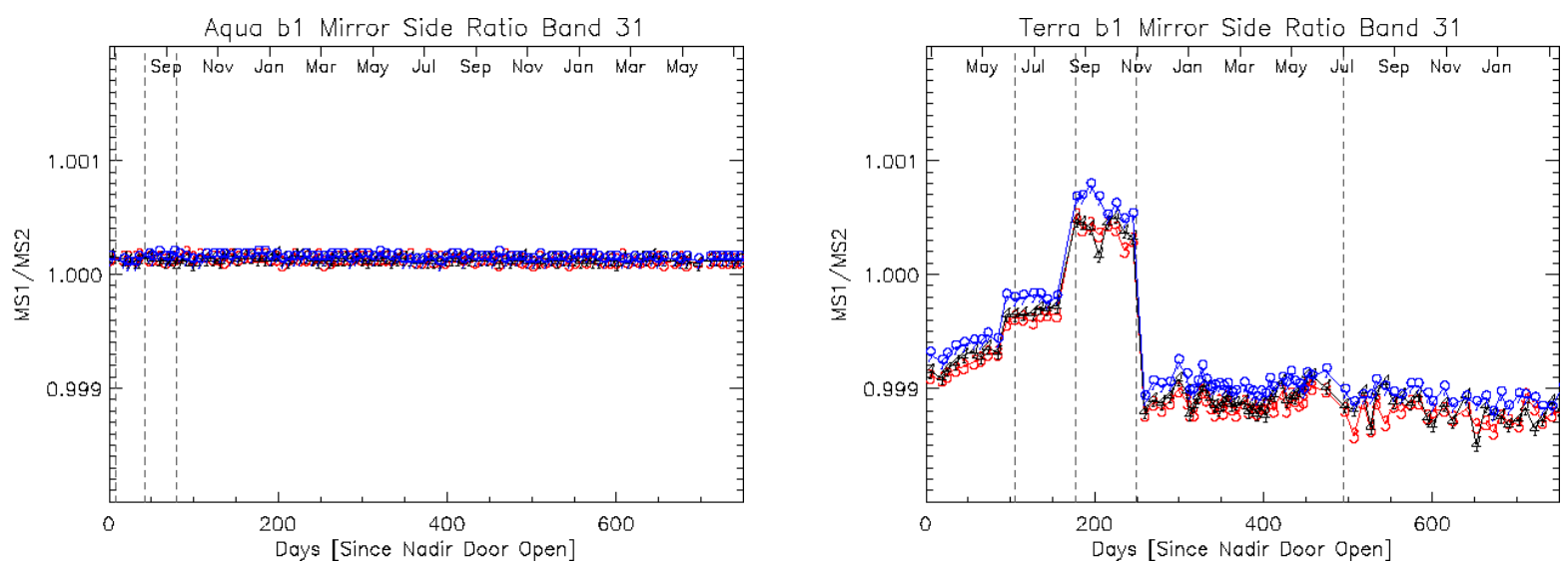

Figure 5: Aqua and Terra MODIS B31 (detectors 3, 4, and 9) mirror side response ratio vs. days. 

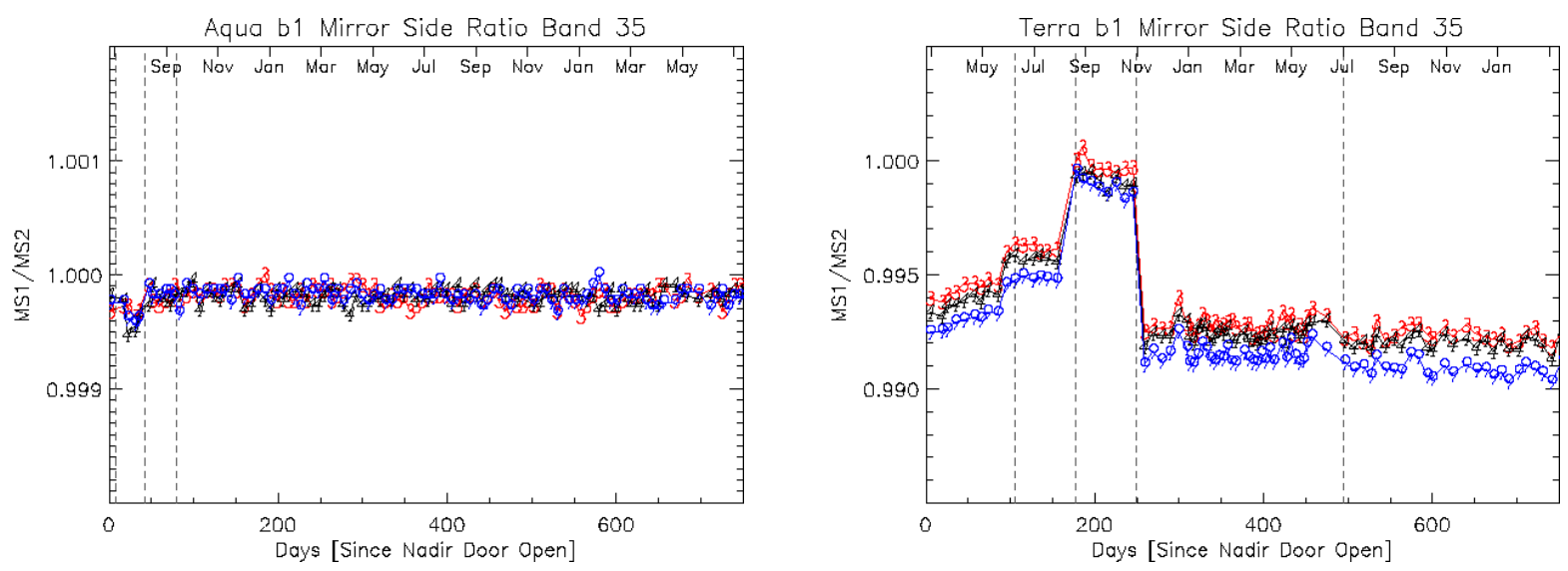

Figure 6: Aqua and Terra MODIS B35 (detectors 3, 4, and 9) mirror side response ratio vs. days.

\subsection{Spectral characterization}

On-orbit spectral characterization (RSB only due to SRCA source and grating limitations) is performance by the SRCA every three months. Observations from the SRCA spectral mode provide information on the relative spectral response and its changes on-orbit and therefore any spectral band center wavelength shifts. The results show that instrument performance in these areas has been very stable and consistent with the pre-launch characterization. There is no obvious difference in the relative spectral response (RSR) functions and the center wavelength shifts on-orbit are less than $0.5 \mathrm{~nm}$ for almost all the reflective solar bands. Figure 7 shows the comparison of center wavelength shifts between Aqua and Terra MODIS bands 3, 8, 9, 10, and 17 during their first two years of on-orbit operation.
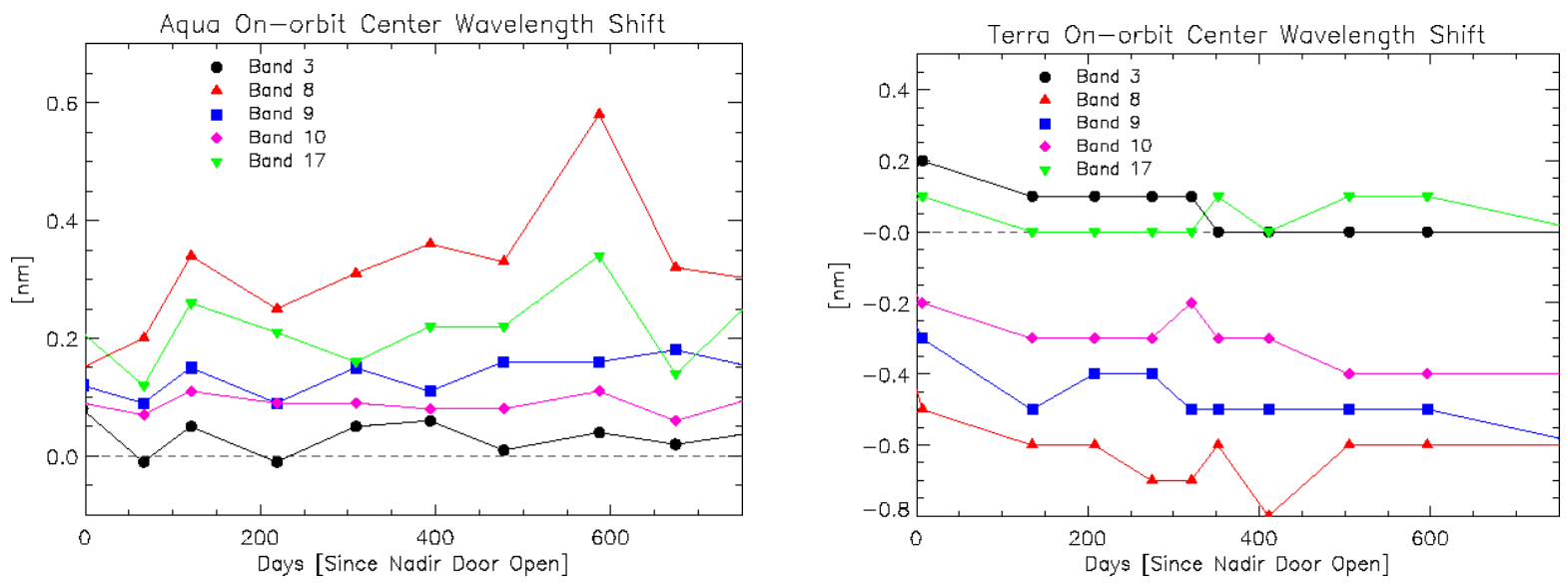

Figure 7: Aqua and Terra MODIS bands 3, 8, 9, 10, and 17 center wavelength shifts vs. days.

4.4 Spatial characterization

On-orbit band-to-band registration (BBR) is determined by the SRCA operated in the spatial mode. The spatial characterization is performed every two months for both the RSB and TEB. Typically the BBR shifts are very small among bands on the same focal plane. Therefore the spatial performance is often evaluated by examining the shifts between different focal planes. Figures 8 and 9 illustrate these shifts for both Aqua and Terra MODIS in the along scan and along track directions.

One of the pre-launch identified problems for the Aqua MODIS was that the instrument's spatial performance was out of specification in band-to-band registration (BBR) and relative shifts among different focal plane assemblies (FPAs), namely between the warm FPAs (VIS and NIR) and the cold FPAs (SMIR and LWIR). This problem appeared in both 
along-scan and along-track BBR and focal plane to focal plane shift. Excluding changes at the beginning of the mission, the spatial performance of Terra MODIS has been much better than Aqua MODIS (note: different scales are used in Figures 8 and 9 for Terra and Aqua BBR). The BBR problem or large shift between different focal planes should be considered when developing science products using bands from different focal planes.
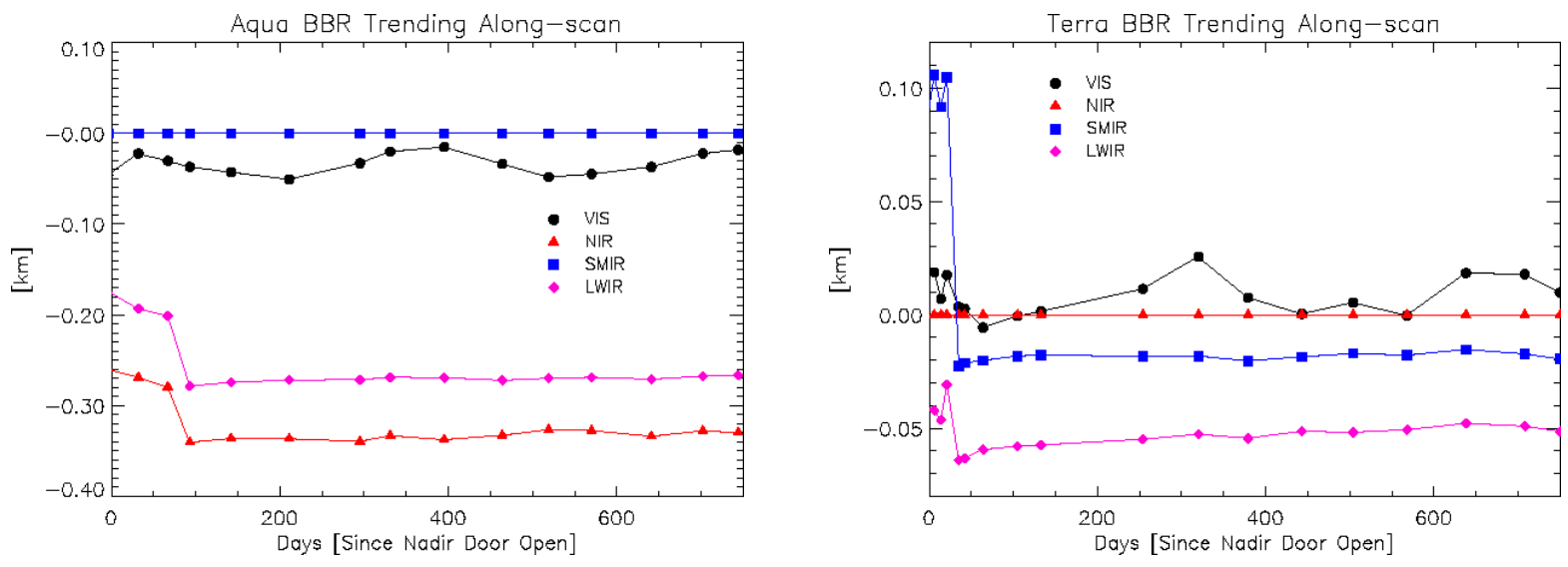

Figure 8: Aqua and Terra focal plane to focal plane shifts vs. days in the along scan direction.
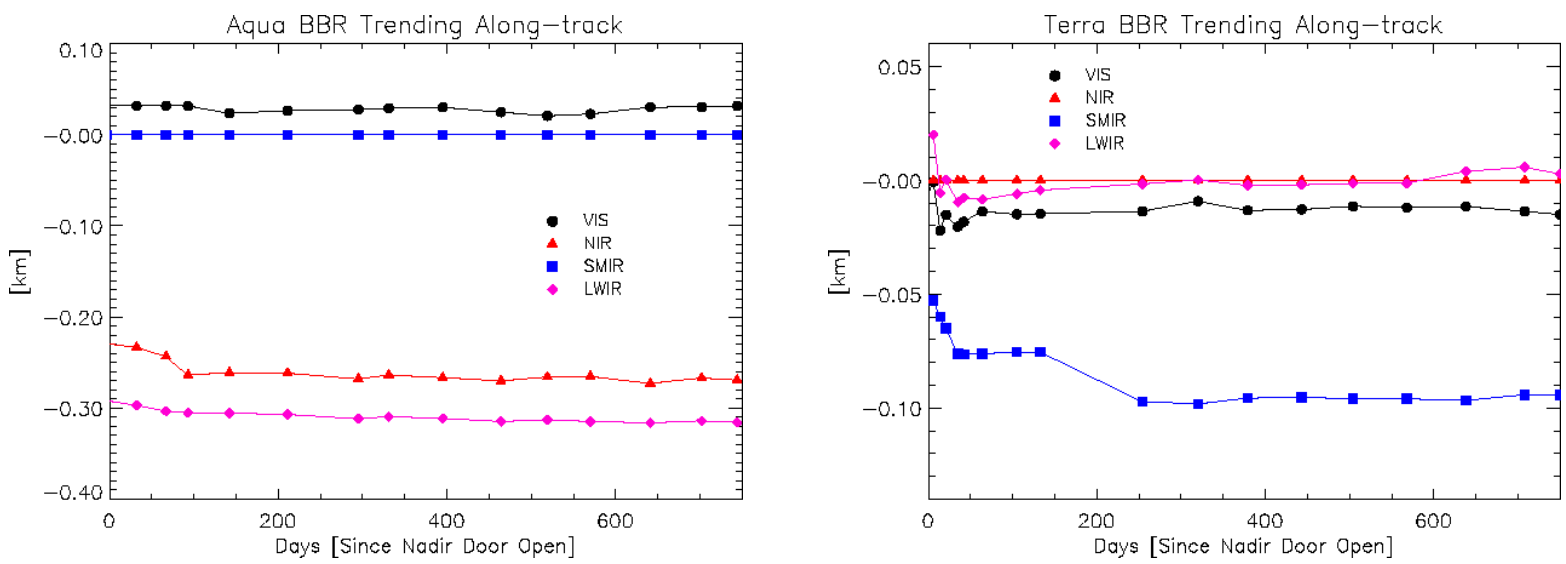

Figure 9: Aqua and Terra focal plane to focal plane shifts vs. days in the along track direction.

\section{SUMMARY}

This paper provides an overview of the MODIS algorithms for on-orbit radiometric calibration and instrument spectral and spatial characterization. It presents the Aqua MODIS instrument's performance during its first two years of on-orbit calibration and characterization and compares it with that from the Terra MODIS in terms of their long term response trending, mirror side differences, center wavelength shifts, and relative spatial shifts between different focal planes. Lessons learned from Terra MODIS have resulted in some improvements in the Aqua MODIS design and test. Early onorbit calibration and characterization experience from Terra MODIS has also played an important role in Aqua MODIS. The overall performance of Aqua MODIS is better than that of Terra MODIS, except for the pre-launch identified problems of B6 detector operability and band-to-band (or focal plane to focal plane) registration and a few on-orbit noisy detectors that do not meet the $\pm 2 \%$ reflectance and $\pm 5 \%$ radiance RSB calibration uncertainty requirements or the $\pm 1 \%$ TEB uncertainty at their specified typical radiance values. On-orbit observations show that the Aqua MODIS is much more stable than the Terra MODIS in a number of areas. The mirror side differences are extremely small and stable for Aqua MODIS compared with Terra MODIS in both the RSB and the TEB. Though on-orbit calibration and characterization of each instrument continue to track and correct these changes, a stable instrument makes it much easier to maintain the calibration and data product quality. 


\section{REFERENCES}

1. W.L. Barnes and V.V. Salomonson, "MODIS: A global image spectroradiometer for the Earth Observing System", Critical Reviews of Optical Science and Technology, CR47, 285-307, 1993

2. W.L. Barnes, V.V. Salomonson, B. Guenther, and X. Xiong, "Development, Characterization, and Performance of the EOS MODIS Sensors", Proceedings of SPIE - Earth Observing Systems VIII, 5151, 2003

3. W.L. Barnes, X. Xiong, and V.V. Salomonson, "Status of Terra MODIS and Aqua MODIS", J. of Advances in Space Research, 32, 2099-2106, 2003

4. C. L. Parkinson, "Aqua: An Earth-Observing Satellite Mission to Examine Water and Other Climate Variables", IEEE Transactions on Geoscience and Remote Sensing, 41, 173-183, 2003

5. C.O. Justice, E. Vermote, J.R.G. Townshend, R. Defries, D.P. Roy, D.K. Hall, V.V. Salomonson, J.L. Privette, G. Riggs, A. Strahler, W. Lucht, R.B. Myneni, P. Lewis, and M.J. Barnsley, "The Moderate Resolution Imaging Spectroradiometer (MODIS): Land Remote Sensing for Global Change Research", IEEE Transactions on Geoscience and Remote Sensing, 36, 1228-1249, 1998

6. W.E. Esaias, M.R. Abbott, I. Barton, O.W. Brown, J.W. Campbell, K.L. Carder, D.K. Clark, R.L. Evans, F.E. Hoge, H.R. Gordon, W.P. Balch, R. Letelier, and P.J. Minnett, "An overview of MODIS capabilities for ocean science observations", IEEE Transactions on Geoscience and Remote Sensing, 36, 1250-1265, 1998

7. M.D. King, W.P. Menzel, Y.J. Kaufman, D. Tanre, B.C. Gao, S. Platnick, S.A. Ackerman, L.A. Remer, R. Pincus, and P.A. Hubanks, "Cloud and aerosol properties, precipitable water, and profiles of temperature and water vapor from MODIS", IEEE Transactions on Geoscience and Remote Sensing, 41, 442-458, 2003

8. V. V. Salomonson, W. L. Barnes, X. Xiong, S. Kempler and E. Masuoka, "An Overview of the Earth Observing System MODIS Instrument and Associated Data Systems Performance", Proceedings of IGARSS, 2002

9. W. L. Barnes, X. Xiong and V.V. Salomonson, "Status of Terra MODIS and Aqua MODIS", Proceedings of IGARSS, 2002

10. X. Xiong and W. L. Barnes, "Early On-orbit Calibration Results from Aqua MODIS," Proceedings of SPIE Sensors, Systems, and Next generation of Satellites VI, 4881, 2002

11. X. Xiong, K. Chiang, J. Sun, N. Che, and W.L. Barnes, "Aqua MODIS First Year On-orbit Calibration and Performance," Proceedings of SPIE - Sensors, Systems, and Next Generation of Satellites VII, 5234, 2003

12. X. Xiong, W.L. Barnes, B. Guenther, and R.E. Murphy, "Lessons Learned from MODIS Calibration and Characterization," J. of Advances in Space Research, 32, 2107-2112, 2003

13. X. Xiong, K. Chiang, W. Li, F. Adimi, H. Yatagai, and W.L. Barnes, "The MODIS Correction Algorithm for Outof-band Response in the Short-wave IR Bands," Proceedings of SPIE - Sensors, Systems, and Next Generation of Satellites VII, 5234, 2003

14. X. Xiong, K. Chiang, J. Esposito, B. Guenther, and W.L. Barnes, "MODIS On-orbit Calibration and Characterization," Metrologia 40 89-92, 2003

15. X. Xiong, J. Sun, J. Esposito, B. Guenther, and W. L. Barnes, "MODIS Reflective Solar Bands Calibration Algorithm and On-orbit Performance", Proceedings of SPIE - Optical Remote Sensing of the Atmosphere and Clouds III, 4891, 2002

16. X. Xiong, K. Chiang, B. Guenther, and W.L. Barnes, "MODIS Thermal Emissive Bands Calibration Algorithm and On-orbit Performance", Proceedings of SPIE - Optical Remote Sensing of the Atmosphere and Clouds III, 4891, 2002

17. H. Montgomery, N. Che, K. Parker, and J. Bowser, "The algorithm for MODIS wavelength on-orbit calibration using the SRCA", IEEE of Geo. And Remote Sensing, Vol. 38, No.2, 877-884, 2002

18. X. Xiong, N. Che, and W.L. Barnes, "On-orbit Spectral Characterization Results for Terra MODIS Reflective Solar Bands", Proceedings of SPIE - Earth Observing Systems VIII, 5151, 2003

19. X. Xiong, N. Che, F. Adimi, and W.L. Barnes, "On-orbit Spatial Characterization for Terra MODIS", Proceedings of SPIE - Earth Observing Systems VII, 4814, 2002

20. H. Montgomery, N. Che, and J. Bowser, "Determination of the spatial characteristic by using the SpectroRadiometric Calibration Assembly (SRCA) of MODIS (Part I. Along-scan)", Proceedings of SPIE - Earth Observing Systems III, 3439, 226-237, 1998

21. H. Montgomery, N. Che, and J. Bowser, "Determination of the spatial characteristic by using the SpectroRadiometric Calibration Assembly (SRCA) of MODIS (Part II. Along-track)", Proceedings of SPIE - Earth Observing Systems III, 3439, 238-246, 1998 